\title{
The Natural History of the Appearance of Apnea of Prematurity
}

\author{
KEITH BARRINGTON AND NEIL FINER \\ Walter Mackenzie Health Sciences Center, Division of Newborn Medicine, University of Alberta Hospitals, \\ Edmonton, Alberta, Canada T6G 2B7 [K.B.] and Division of Newborn Medicine, Royal Alexandra Hospitals,
} Edmonton, Alberta, Canada T5H 2 V9 [N.F.]

\begin{abstract}
Twenty healthy preterm infants of less than 34 wk gestation were studied with continuous recordings, commencing within $8 \mathrm{~h}$ of birth, for up to 1 wk of age to determine the usual time course of the appearance of apnea and to classify apnea types. Airway occlusion studies were also performed on a regular basis to determine whether apneic spells were preceded or followed by a reduction in central respiratory drive. Apneic spells of greater than $\mathbf{1 5}$ $s$ duration accompanied by hypoxia or bradycardia occurred in all infants before $24 \mathrm{~h}$ of age. The frequency of apneic spells was highest in the first $24 \mathrm{~h}$ after birth with a mean frequency of $0.9 / \mathrm{h}$ and gradually reduced thereafter, falling to $0.2 / \mathrm{h}$ by $5 \mathrm{~d}$ of age $(p<0.01)$. Apneic spells were more likely to be obstructive in the first $2 \mathrm{~d}$ of life than thereafter $(p<0.05)$. Central apnea was proportionately significantly less frequent during this time period. Reduced respiratory drive, as demonstrated by airway occlusion pressures, was associated with more frequent apnea and was evident at the first occlusion study, which frequently preceded the first significant apnea. (Pediatr Res 29: 372-375, 1991)
\end{abstract}

\section{Abbreviations}

$\mathrm{SpO}_{2}$, pulse oximeter-derived saturation

$P_{0.1}$, airway pressure $0.1 \mathrm{~s}$ after initiation of first occluded breath

ANOVA, analysis of variance

It is commonly stated that idiopathic apnea of prematurity develops after the first 24 to $72 \mathrm{~h}$ of life and apnea that is noted early requires vigorous investigation (1). The factual basis of this statement is uncertain. Henderson-Smart (2) previously demonstrated that apnea (more than three spells of greater than $20 \mathrm{~s}$ duration) developed on the 1 st or 2 nd postnatal $d$ in $77 \%$ of preterm infants. He appears to have used impedance monitoring only and it is uncertain whether any recordings were made for independent confirmation of apnea (3). Carlo et al. (4), utilizing 60 -min recordings, showed that apneic spells of greater than $5 \mathrm{~s}$ duration occurred on $d 1$ in eight of 10 preterm infants without lung disease.

There appears to be no published information regarding the usual frequency and nature of significant apneic spells in an unselected population of healthy newborn preterm infants who have undergone prolonged recordings.

Our main objective was to ascertain the usual age of the

Received March 28, 1990; accepted December 5, 1990.

Reprint requests: Dr. K. Barrington, 3A3 Walter Mackenzie Health Sciences Center, Division of Newborn Medicine, University of Alberta Hospitals, Edmonton, Alberta, Canada T6G 2B7.

Supported by the Northern Alberta Children's Hospital Foundation. appearance of apneic spells and the relative frequency of obstructive, mixed, and central apnea during the $1 \mathrm{st}$ wk of life in preterm infants without respiratory disease. Our secondary objective was to describe the development of respiratory drive, by determining the postnatal progression of occlusion pressures, in infants with and without apnea.

\section{SUBJECTS AND METHODS}

Twenty infants of less than 34 wk gestation with birth weights above the 5 th percentile for gestational age were studied. Infants were all born in the Royal Alexandra Hospitals and were entered into the study as soon after birth as possible. We entered consecutive infants who were clinically determined to be free of acute medical problems; in particular, they were without significant respiratory disease. The criteria for this were an oxygen requirement of no more than $30 \%$, respiratory rate less than $70 / \mathrm{min}$, and no clinical suspicion of pneumonia or hyaline membrane disease. Infants requiring any supplemental oxygen had chest radiographs, which were required to be normal. Ultrasound examination of the head was performed in all infants before $4 \mathrm{~d}$ of age and again at approximately 2 wk of age. Any infant with intraventricular hemorrhage that distended the ventricles or evidence of intracerebral hemorrhage or periventricular leukomalacia on either examination was eliminated from the study.

After the initial clinical examination and assessment to ensure that the infants were stable, each was monitored with a combination of impedance pneumogram, for the detection of chest wall movement, ECG, pulse oximetry $\left(\mathrm{SpO}_{2}\right)$, and end-tidal $\mathrm{CO}_{2}$ monitoring; some infants were monitored with transcutaneous $\mathrm{PO}_{2}$ in addition. The analogue outputs of each of these monitors was digitized by a Data-translation DT2800 analogue to digital convertor board (Data Translation, Inc., Marlborough, MA) and then analyzed on a Compaq 286 personal computer (Compaq Computer Corp., Houston, TX), using programs that we wrote using the Asyst programming language (Asyst Software Industries, Rochdale, NY).

At this point, parental consent was sought for the airway occlusion studies and for the continuance of noninvasive monitoring. In the event of parental refusal of the occlusions, consent for continued monitoring alone was requested. This protocol was approved by the clinical investigation committee of the Royal Alexandra Hospitals.

After obtaining consent, airway occlusions were performed as previously described (5). A face mask was applied and the absence of leaks was confirmed. Airflow was monitored by means of a Fleisch 00 pneumotachograph and integrated electronically to give tidal volume. At end-expiration the airway was occluded and during the next breath the resultant airway pressures were recorded. The $P_{0.1}$ was recorded. Three occlusion responses were obtained on each occasion and averaged. This process was performed as soon as possible after birth and then every $12 \mathrm{~h}$ for 
the next $72 \mathrm{~h}$. Thereafter, occlusions were performed daily until the study terminated.

The study continued until the infant was 1 wk old or until therapy (theophylline) for idiopathic apnea of prematurity was prescribed by the attending physician. Thus, all of the results presented herein are from infants who were not receiving methylxanthines or any other therapy for apnea. The clinical staff had no knowledge of the results of the recordings and clinical diagnosis of apnea was according to the usual unit practice of examination of the nursing records.

At the termination of the study, the recordings were analyzed by means of a semiautomated process (6). The program used will flag an epoch that is considered to contain a potential apneic spell by looking for a combination of any two of the following: loss of end-tidal $\mathrm{CO}_{2}$, fall in heart rate, and fall in $\mathrm{SpO}_{2}$. The epoch may then be visually inspected to determine whether a significant apneic spell was present. The criteria for the diagnosis of a significant apneic spell were as follows: lack of nasal airflow for $15 \mathrm{~s}$ accompanied by either a fall in heart rate $20 \%$ below the previous mean heart rate or a fall in $\mathrm{SpO}_{2}$ of 10 percentage points. When a spell was identified, its duration and time of occurrence were noted and it was classified into central, obstructive, or mixed. Central spells were those in which there were no obstructed breaths during the apnea. The presence of one or more obstructed respiratory efforts prompted the diagnosis of a mixed or obstructive apneic spell, which were further differentiated by the presence of a period of absent chest wall movement of at least 3 to $4 \mathrm{~s}$ duration.

Apnea frequency was calculated on a daily basis. For the first $24 \mathrm{~h}$, the apnea frequency was calculated as the number of apneas noted before $24 \mathrm{~h}$ of age divided by the number of hours of recording performed. Thereafter, the apnea frequency was calculated by dividing the daily number of apneas by 24 .

Statistical methods included a two-way ANOVA to compare frequencies of apnea with respect to apnea types and postnatal age, with a protected Fisher's least significant difference being used as the post hoc test to define intergroup differences in the event of a significant ANOVA. Pearson correlation coefficient was used to determine the significance of the relationship between apnea frequency and occlusion pressures.

Some data from 10 of these infants has been previously reported (6) (incidence of periodic breathing and relationship between periodicity and apnea).

\section{RESULTS}

The 20 infants were studied commencing at a mean age of 3.8 h (range 2 to 8 ). Their mean birth weight was $1012 \mathrm{~g}$ (SD 322) and mean gestation was $29.7 \mathrm{wk}$ (SD 2.7). Thirteen infants were delivered by the vaginal route and seven by cesarean section. The first head ultrasound revealed six infants with subependymal hemorrhages. Two of these infants progressed to small intraventricular hemorrhages. Three infants required supplemental oxygen when first entered into the study to maintain an $\mathrm{SpO}_{2}$ of greater than $92 \%$; all were in room air by $12 \mathrm{~h}$ of age.

All infants in the study experienced significant apneic spells before $24 \mathrm{~h}$ of age. The mean age of the first recorded apnea was $8.5 \mathrm{~h}$ (SD 3.6, range 3 to 18 ), which was a mean of $4.7 \mathrm{~h}$ (SD 4.0 ) after initiating the recording. The daily distribution of apneic spells is shown in Figure 1. When the daily apnea rates were compared, there was a significant difference in total apnea frequency between $\mathrm{d} 1$ and $\mathrm{d} 4$ to 7 (ANOVA, $F_{6,133}=3.2, p<$ $0.01)$ Table 1 . Obstructive and mixed apneas were significantly more frequent on $\mathrm{d} 1$ than on $\mathrm{d} 4$ to $7(p<0.05)$. Central apneic spells were not significantly different in frequency between any $2 \mathrm{~d}$; thus, the proportions of types of apneic spells were significantly different between $\mathrm{d} 1$ and $\mathrm{d} 4$ to $7\left(\chi^{2} p<0.05\right)$.

Thirteen of the infants were diagnosed as having clinical apnea during the 1 st wk of life and all were treated with theophylline. Seven of the infants therefore had recordings for the entire 1st wk of life. None of the infants were removed from the study before $\mathrm{d} 4$, three were excluded on $\mathrm{d} 5$, seven on $\mathrm{d} 6$, and three on $\mathrm{d} 7$. There were no significant differences in apnea frequency or apnea subtype distribution between $\mathrm{d} 4$ and $\mathrm{d} 7$.

We wished to determine whether there was a threshold number, or duration, of apneic spells that would trigger clinical recognition of apnea and subsequent treatment. On $\mathrm{d} 4,5$, and 6 of the study there were, therefore, a group of infants who received therapy the next day (and were then removed from the study) and a group of infants that were not treated next day. On each of the $3 \mathrm{~d}$, the "not treated next day" infants included the seven who never received therapy (Table 2). Apnea frequency was therefore compared between the groups, using a $t$ test with Bonferroni correction. There were no significant differences on d 4,5 , or 6 . Eight of the 13 treated infants had a work up for sepsis that included a complete blood count, blood culture, and spinal tap. All cultures were negative.

Airway occlusion studies were performed in 16 infants. The first study was performed at a mean postnatal age of $7 \mathrm{~h}$ and preceded the first recorded apnea in 12 of the infants. There was a progressive nonsignificant increase in the $P_{0.1}$ from the first to the last day of the study. There was a significant negative association $(r=-0.57, p<0.05)$ between the apnea frequency over the first three days and $P_{0.1}$ measured at the time of the first occlusion study, but a very wide scatter prevents this from being a useful predictive test (Fig. 2).

\section{DISCUSSION}

There was a $100 \%$ incidence of significant apnea in this study during the first $24 \mathrm{~h}$ of life. This contrasts with studies that used either brief (4) recordings or none at all (2) and emphasizes the importance of both continuous recordings and airflow monitoring for studies on apnea. Our findings imply that there is a $95 \%$ probability that $85 \%$ or more of clinically healthy infants of less than 34 wk gestation will have apneic spells on the first day.

We have performed a number of comparisons of our unique, purpose written programs with paper recordings at each state of development. These evaluations have demonstrated that significant apneic spells are not missed and many artefacts that a fully automated method could record as apneas are eliminated. The use of a single nasal detector could lead to problems if mouth opening or nasal blockage occurred; however, the requirement for hypoxia or bradycardia, in addition to the loss of nasal airflow, to diagnose an apnea makes this an unlikely cause for confounding of our results.

There is no generally accepted definition of what constitutes a significant apneic spell; investigators in recent years have accepted spells of $10(7), 15(8,9), 20(10)$, or $30(11)$ s duration regardless of the occurrence of other physiologic changes. It is also frequently stated that shorter apneas were considered to be significant if associated with either hypoxia or bradycardia. Our definition of apnea requires both a duration of at least $15 \mathrm{~s}$ and either hypoxia or bradycardia. If we had simply required a duration of $15 \mathrm{~s}$, regardless of hypoxia or bradycardia, we would have reported an even higher incidence of early apneic spells.

The initial objective of the study was to compare the early occlusion pressures of infants who developed apnea to control infants who did not. However, in view of the absence of nonapneic infants, we revised our planned analysis of the results. We demonstrated that occlusion pressures were lower in the infants with the most frequent apneic spells before the occurrence of the first recorded apnea. This suggests that apneic spells and reduced occlusion pressures may be caused by similar factors. Previous data from adults indicate that reduced respiratory drive may occur as a result of multiple apneic episodes, because respiratory drive gradually improves after tracheostomy $(12,13)$. Our data would not support the idea that occlusion pressures in premature infants are reduced secondarily to the physiologic effects of apneic spells. 

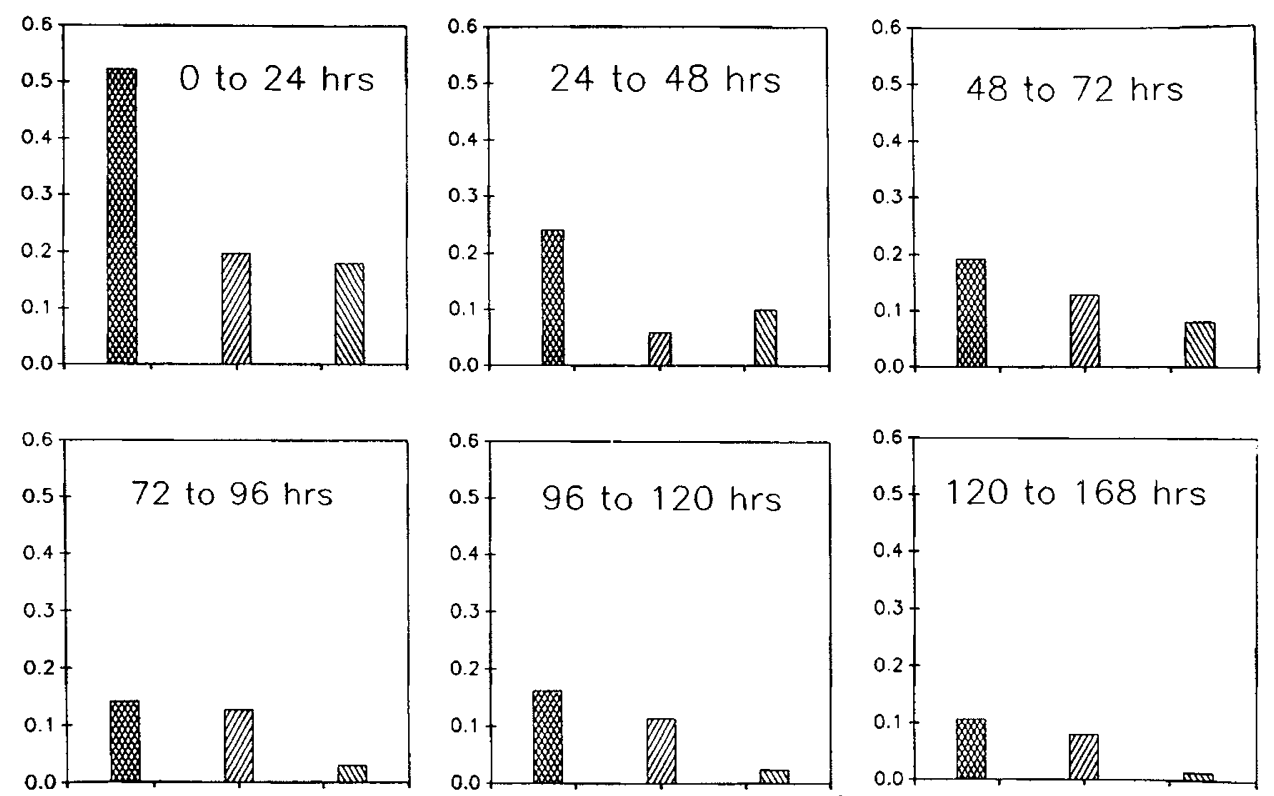

Fig. 1. Apnea frequency $(/ \mathrm{h})$ during consecutive periods after birth in 20 premature infants. Mixed apneas obstructive apneas $\mathbb{\mathbb { N }}$, and central apneas

Table 1. Mean and range of daily total apnea frequencies and apnea durations

\begin{tabular}{|c|c|c|c|c|c|c|c|}
\hline & \multicolumn{7}{|c|}{ Day } \\
\hline & 1 & 2 & 3 & 4 & 5 & 6 & 7 \\
\hline Apneas $>15 \mathrm{~s}(/ \mathrm{h})$ & 0.9 & 0.4 & 0.4 & 0.3 & 0.3 & 0.3 & 0.2 \\
\hline Maximum frequency $(/ \mathrm{h})$ & 2.1 & 2.5 & 1.6 & 2.6 & 1.1 & 1.8 & 1.7 \\
\hline Minimum frequency $(/ \mathrm{h})$ & 0.21 & 0.13 & 0.08 & 0.04 & 0.08 & 0.08 & 0.04 \\
\hline Mean duration (s) & 24.2 & 25.4 & 25.4 & 25.7 & 29.8 & 28.5 & 24.2 \\
\hline Maximum duration (s) & 49.1 & 68.2 & 50.0 & 43.2 & 49.5 & 53.1 & 32.0 \\
\hline Apneas $>20 \mathrm{~s}(/ \mathrm{h})$ & 0.6 & 0.3 & 0.2 & 0.2 & 0.1 & 0.2 & 0.1 \\
\hline
\end{tabular}

Table 2. Apnea frequency $(/ h)$ of infants treated and not treated for apnea of prematurity

\begin{tabular}{|c|c|c|c|c|c|c|c|c|c|}
\hline & \multicolumn{3}{|c|}{ Day 4} & \multicolumn{3}{|c|}{ Day 5} & \multicolumn{3}{|c|}{ Day 6} \\
\hline & $n$ & Mean & $\mathrm{SD}$ & $n$ & Mean & SD & $n$ & Mean & SD \\
\hline Treated next day & 3 & 0.47 & 0.23 & 7 & 0.35 & 0.19 & 3 & 0.24 & 0.22 \\
\hline Not treated next day & 17 & 0.26 & 0.20 & 10 & 0.28 & 0.21 & 7 & 0.31 & 0.11 \\
\hline Never treated & 7 & 0.32 & 0.14 & 7 & 0.29 & 0.18 & & & \\
\hline
\end{tabular}

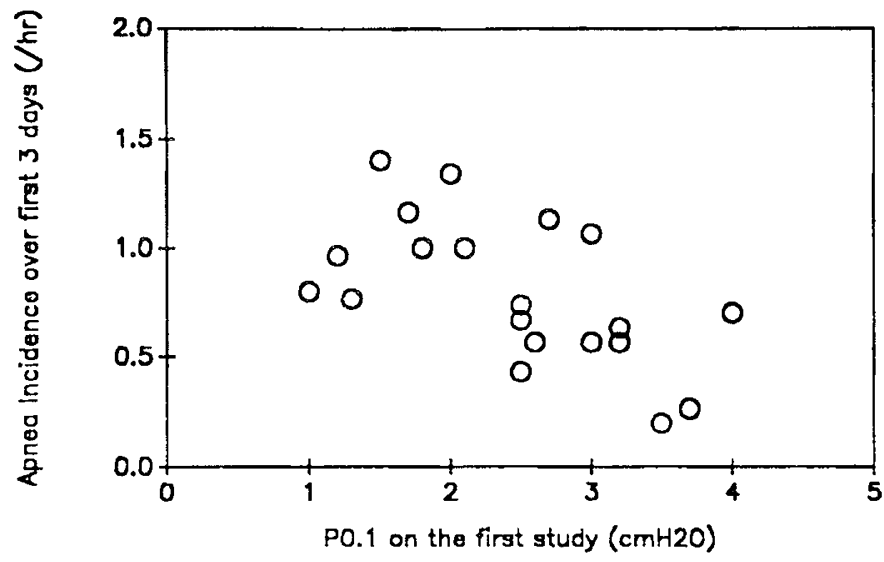

Fig. 2. Relationship between occlusion pressure $\left(\mathrm{P}_{0.1}\right)$ and apnea frequency during the first $3 \mathrm{~d}$ of life.
Airway occlusion pressures, although commonly used as an index of respiratory center output, also reflect the state of contractility of the respiratory muscles (14); the association between occlusion pressure and apnea severity could, therefore, be due to an effect of respiratory muscle contractility. Indeed, there may well be an association between fatigue of the diaphragm and apneic spells (15). We currently have no data to confirm or refute this suggestion. There is also little data on the effects of chest wall distortion on perceived occlusion pressures. It seems likely that chest wall distortion decreases measured occlusion pressures and may also be related to apneic spells because of an increase in respiratory work (16). We did not measure chest wall movement or distortion during occlusion studies.

Our finding of early apnea confirms our previous report (6) and the suggestions of Henderson-Smart (2) and Carlo et al. (4). The common assertion that idiopathic apnea of prematurity occurs later may be due to the use of thoracic impedance as the exclusive method of clinical apnea monitoring in neonatal nurseries. That is a method that will not detect obstructive apneas or the obstructive portion of mixed apneas. To detect the frequent obstructed breaths of preterm infants, a monitor of oronasal airflow, such as the nasal detection of expired $\mathrm{CO}_{2}$, is required.

The frequency of early apnea suggests that deficient immediate postnatal adaptation is etiologically related to the spells. We postulate that reduced central respiratory drive is associated with both reduced occlusion pressures and apneic spells. Central respiratory drive is dependent upon afferent input from a number of sources (17). We suggest that the documented suppression of the peripheral chemoreceptor during this interval, because of the sudden increase in arterial $\mathrm{PO}_{2}(18,19)$, could well be a cause of both reduced respiratory drive and obstructive apneas. Two 
convergent lines of evidence support this suggestion. First, arousal is required for termination of apneic spells in adults with obstructive sleep apnea (13). Activity of the carotid bodies is required for arousal in adult dogs (20) and newborn lambs (21) subjected to progressive hypoxia. Thus, carotid body inactivity could well lead to failure of arousal and to apneas becoming prolonged and more likely to be associated with hypoxia and bradycardia.

Second, the peripheral chemoreceptors appear to be particularly important in the maintenance of upper airway muscle activity. Evidence from both adult animals (22) and newborn infants (23) supports this and would suggest that during immediate postnatal adaptation maintenance of upper airway patency would be less efficient and obstructive and mixed apnea would be more common.

We were unable to demonstrate any significant differences between infants who were removed from the study because they were treated for apnea with methylxanthines and those who remained untreated. The clinical staff was not aware of the results of the computer analysis and the question of what prompted the clinicians to treat apnea remains unresolved. We assume that either the reporting of the apneic spells by the bedside nurse to the physician may have differed or the threshold that individual physicians used to treat apnea may have varied. Because of the lack of agreement concerning the outcome of infants who experience apnea of prematurity $(24,25)$, it is inevitable that such differences will persist. Further research is required to determine what severity, frequency, and type of apneic spells are associated with clinically significant impairment.

\section{REFERENCES}

1. Vyas H, Milner AD 1986 Other respiratory disorders in the neonate. In Roberton NRC (ed) Neonatology. Churchill Livingstone, Edinburgh, pp 312-339

2. Henderson-Smart DJ 1981 The effect of gestational age on the incidence and duration of recurrent apnoea in newborn babies. Aust Paediatr J 17:273276

3. Muttit S, Finer NN, Tierney AJ, Rossmann J 1988 Neonatal apnea: diagnosis by nurse versus computer. Pediatrics $82: 713-720$

4. Carlo WA, Martin RJ, Versteegh FGA, Goldman MD, Robertson SS, Fanaroff AA 1982 The effect of respiratory distress syndrome on chest wall movement and respiratory pauses in preterm infants. Am Rev Respir Dis 126:103-107

5. Barrington KJ, Finer NN, Peters KL, Barton J 1986 The physiologic effects of doxapram in idiopathic apnea of prematurity. J Pediatr 108:124-129
6. Barrington KJ, Finer NN 1990 Periodic breathing and apnea in preterm infants. Pediatr Res 27:118-121

7. Jones RAK 1982 Apnoea of immaturity. 1. Controlled trial of theophylline and face mask continuous positive airways pressure. Arch Dis Child 57:761765

8. Hiatt IM, Hegyi T, Indyk L, Dangman BC, James LS 1981 Continuous monitoring of $\mathrm{PO}_{2}$ during apnea of prematurity. J Pediatr 98:288-291

9. Boros SJ, Reynolds JW 1976 Prolonged apnea of prematurity: treatment with continuous distending pressure delivered by nasopharyngeal tube. Clin $\mathrm{Pe}$ diatr (Phila) 15:123-134

10. Gerhardt T, Bancalari E 1984 Apnea of prematurity: 1. Lung function and regulation of breathing. Pediatrics 74:58-62

11. Lagercrantz H, Rane A, Tunell R 1980 Plasma concentration-effect relationship of theophylline in treatment of apnea in preterm infants. Eur $\mathrm{J}$ Clin Pharmacol 18:65-68

12. Guilleminault C, Cummiskey J 1982 Progressive improvement of apnea index and ventilatory response to $\mathrm{CO}_{2}$ after tracheostomy in obstructive sleep apnea syndrome. Am Rev Respir Dis 126:14-20

13. Bradley TD, Phillipson EA 1985 Pathogenesis and pathophysiology of the obstructive sleep apnea syndrome. Med Clin North Am 69:1169-1185

14. Aubier M, Murciano D, Viires N, Lecoguic Y, Palcios S, Pariente R 1983 Increased ventilation caused by improved diaphragmatic efficiency during aminophylline infusion. Am Rev Respir Dis 127:148-154

15. Lopes JM, Muller NL, Bryan MH, Bryan AC 1981 Synergistic behavior of inspiratory muscles after diaphragmatic fatigue in the newborn. $J$ Appl Physiol 51:547-551

16. Guslits BG, Gaston SE, Bryan MH, England SJ, Bryan AC 1987 Diaphragmatic work of breathing in premature human infants. I Appl Physiol 62:14101415

17. Sullivan CE, Kozar LF, Murphy E, Phillipson EA 1978 Primary role of respiratory afferents in sustaining breathing rhythm. J Appl Physiol 45:1117

18. Belenky DA, Standaert TA, Woodrume DE 1979 Maturation of hypoxic ventilatory response of the newborn lamb. J Appl Physiol 47:927-930

19. Hertzberg T, Lagercrantz H 1987 Postnatal sensitivity of the peripheral chemoreceptors in newborn infants. Arch Dis Child 62:1238-124

20. Bowes G, Townsend ER, Kozar LF, Bromley SM, Phillipson EA 1981 Effect of carotid body denervation on arousal response to hypoxia in sleeping dogs. J Appl Physiol 51:40-45

21. Fewell JE, Kondo CS, Dascalu V, Filyk SC 1989 Influence of carotid denervation on the arousal and cardiopulmonary response to rapidly developing hypoxaemia in lambs. Pediatr Res 25:473-477

22. Bruce EN, Mitra J, Cherniack NS 1982 Central and peripheral chemoreceptor inputs to phrenic and hypoglossal motoneurones. J Appl Physiol 53:15041511

23. Carlo WA, DiFiore JM, Martin RJ 1989 Increased upper airway muscle activity during hypoxaemia-induced respiratory depression in preterm infants. Pediatr Res 25:373A(abstr)

24. Jones RAK, Lukeman D 1982 Apnoea of immaturity. 2. Mortality and handicap. Arch Dis Child 57:766-768

25. Levitt GA, Mushin A, Bellman S, Harvey DR 1988 Outcome of preterm infants who suffered neonatal apneic attacks. Early Hum Dev 16:235-243 